\title{
Focused Ion Beam Enabled Analysis of Nanocomposite and Nanostructured Soft Materials
}

\author{
S.E. Kooi,* J.-H. Jang,* C. Ullal,** and E.L. Thomas** \\ * Institute for Soldier Nanotechnologies, Massachusetts Institute of Technology, Cambridge, MA \\ 02139 \\ ** Department of Materials Science, Massachusetts Institute of Technology, Cambridge, MA 02139
}

Structural and compositional analysis of nanocomposite/nanostructured materials by transmission electron microscopy (TEM) and atomic force microscopy (AFM) methods are often limited due to sample preparation issues. In the case of nanocomposite materials composed of hard and soft domains, such as carbon nanotubes or clay platelets dispersed in a polymer matrix or a soft polymer film on a hard substrate, standard sample preparation techniques (microtoming) often fail to produce representative samples that are thin enough to be electron transparent for TEM or smooth enough for AFM. We have adapted focused ion beam (FIB) techniques, which are more typically used for semiconductor and metallic materials, to produce both electron transparent and smooth samples from these types of nanocomposite materials. Results will be presented on carbon nanotubes and nanoparticle polymer composites as well as polymer films on structured silicon substrates.

FIB techniques have also been applied to characterize three dimensionally structured polymeric materials produced by multibeam laser interference lithography[1]. These 3D structured polymers have potential as photonic and phononic band gap materials as well as lightweight energy absorbing materials. The properties of these materials are highly dependent on the quality of their three dimensional structure. We have used a FIB to produce multiple consecutive cross sectional images of these materials and have used the images to reconstruct the overall 3D structure produced. [2]

\section{References}

[1] C. Ullal et al., Appl. Phys. Lett. 84 (2004) 5434.

[2] This research was supported by the US Army through the Institute for Soldier Nanotechnologies, under Contract DAAD-19-02-D-0002 with the US Army Research Office. The content does not necessarily reflect the position of the Government, and no official endorsement should be inferred. 

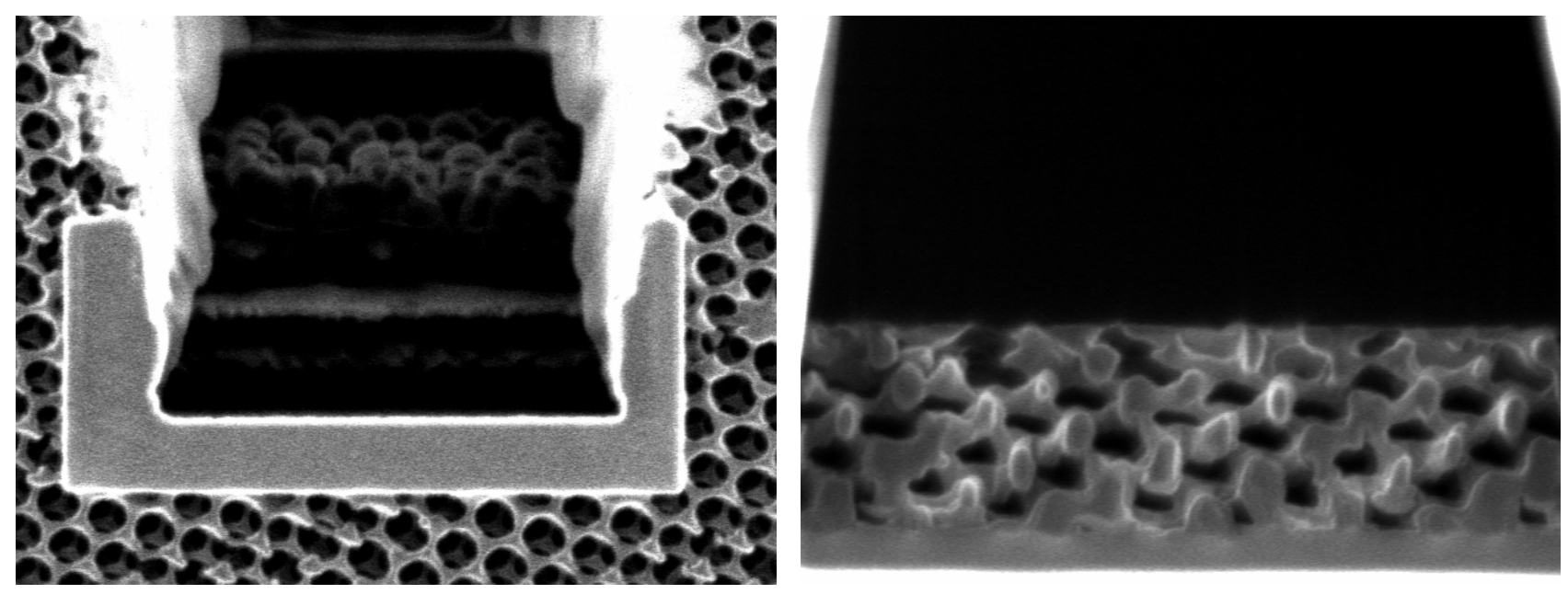

FIG. 1. Secondary electron images of an interference lithography produced structure. The left image shows a top down view of the area being sectioned in a FIB and the right image shows the cross sectioned face of the structure at a 50 degree tilt angle. 